\title{
Smart Dust Bin With Automatic Lid and Buzzer Alarm
}

\author{
Aida Mahdalena Lubis, Fabio Sarmento Freitas Guterres, Joana Victorine Harryanto, Mia Galina \\ Department of Electrical Engineering, President University, Bekasi 17550, Indonesia \\ Email: aidamahda579@gmail.com,guterresfabio44@gmail.com,vtrn.jo@gmail.com, miagalina@president.ac.id \\ Corresponding author: aidamahda579@gmail.com
}

\begin{abstract}
Touching dustbins by hand often are problematic by some people due to questionable hygiene conditions. This, along with laziness to dispose garbage properly may also be a factor that makes people hesitate to dispose of garbage. This project aims to make use of microcontroller technology to create a Smart Dust Bin. This prototype would have a lid that automatically open when a hand is approaching it which eliminates the need to physically touch the bin. The device also has internal alarm system which will sound whenever the content reaches level high enough inside the bin. Upon a test, these two features work properly.
\end{abstract}

Keywords: Dustbin, automatic, alarm, smart.

\section{Introduction}

Nowadays, technology has continued to advance and help to ease the daily activities and work of human beings. People continue to research and try to improve the technology to be used in their work to create an allautomatic system. Especially with the help of the internet, everything is slowly shifting to automatic devices where humans no longer need to do things manually.

Using Arduino as a microcontroller and some knowledge that has been gained from Intro to Embedded System subject, we have created a simple but powerful system that could help people improve the new traditional dustbin in a more sophisticated and automatic way.

This research aims to create a garbage can with a lid that can be automatically opened and closed and an automatic alarm when the dustbin is full using two ultrasonic sensor HC-SR04, Servo, and a Buzzer. With the presence of this bin, it is expected that it will raise the community's interest in disposing of the garbage without fear of the bacteria attached to the trash, as it is not necessary for the need to be accessed to open it.

\section{System Design}

\section{A. Circuit Diagram}

The prototype of this project uses Arduino Uno as the main controller, one Buzzer as the alarm when the content of the bin reaches certain height, one ultrasonic sensor to be placed outside to detect user's hand and trigger the servo to move the lid, one ultrasonic sensor inside the bin to detect the bin content's height and trigger the buzzer, and one servo to control the opening and closing the lid. The circuit connection is shown in Figure 1.

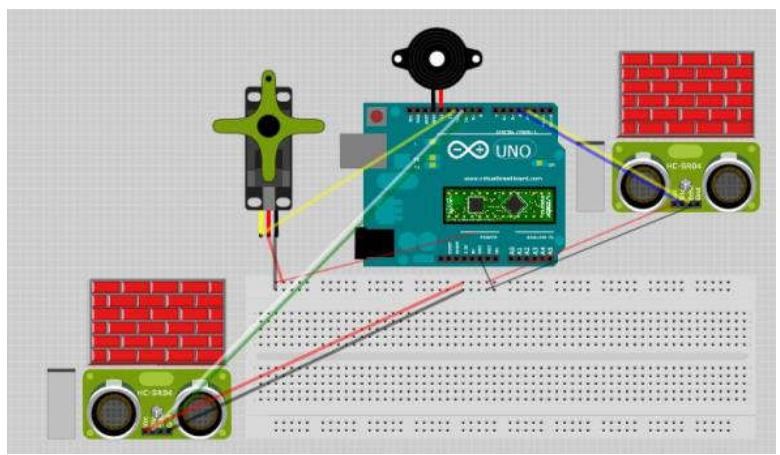

Figure 1. System Circuit Diagram.

The ultrasonic sensor used in this project is HC-SR04 while TowerPro SG-05 is used as the servo. This servo has pretty low torque, but in this project, it is enough as the material for the lid to be lifted by the servo does not require high torque.

The connections for the first ultrasonic sensor (to control servo) and the second ultrasonic sensor (to trigger buzzer) can be seen in Table 1 and Table 2, respectively. Meanwhile, the connection of the servo is shown in Table 3.

Table 1. Ultrasonic sensor for Servo Connection

\begin{tabular}{|l|l|}
\hline VCC & $5 \mathrm{~V}$ \\
\hline GND & Ground \\
\hline Trigger Pin & Pin \#10 of Arduino \\
\hline Echo Pin & Pin \#9 of Arduino \\
\hline
\end{tabular}

Table 2. Ultrasonic sensor for Buzzer Connection

\begin{tabular}{|l|l|}
\hline VCC & $5 \mathrm{~V}$ \\
\hline GND & Ground \\
\hline Trigger Pin & Pin \#4of Arduino \\
\hline Echo Pin & Pin \#3 of Arduino \\
\hline
\end{tabular}


Table 3. Servo Motor Connection

\begin{tabular}{|l|l|}
\hline Pin 1 & Pin \#11 of Arduino \\
\hline Pin 2 & VCC \\
\hline Pin 3 & Ground \\
\hline
\end{tabular}

The final look of the circuit before being rewired into the dustbin is shown in Figure 2.

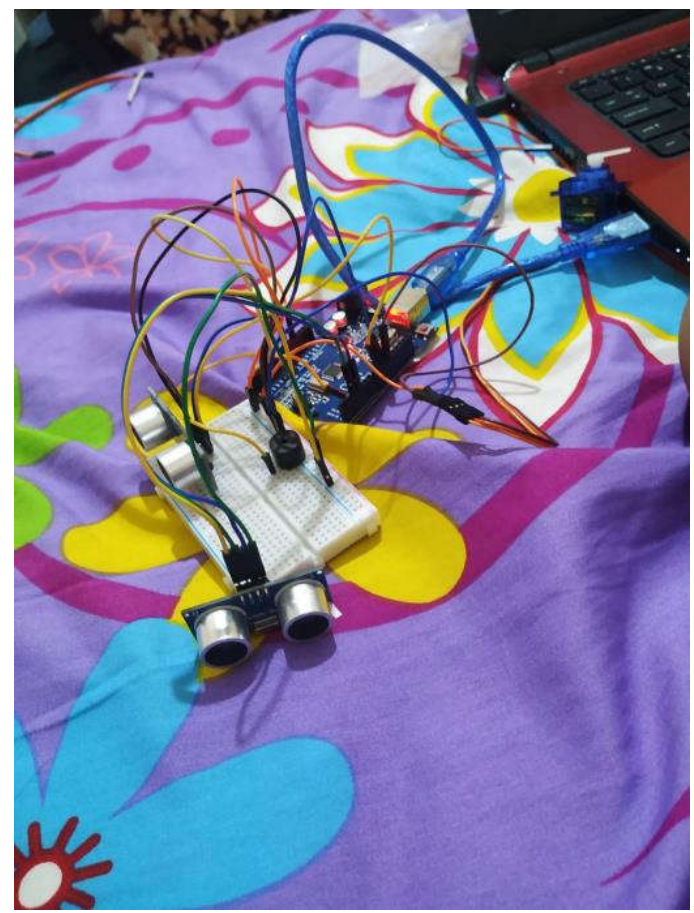

Figure 2. Final Circuit before being integrated into the bin.

\section{B. The DustBin}

The bin used in this prototype was commonly sold in the market. The lid, however, needs to be made custom to the project. The lid is created using light material called impra-board. The design of the lid is as follows:

1. Create a circle with the impra-board with a diameter according to the diameter of the cover. \# Make sure the diameter is not the EXACT value of the cover, because we need the cover to be able to close the bin at the top.

2. Cut the ring into $3 / 4$ (See Figure 2, which will serve as an opener to be pulled by a servo.

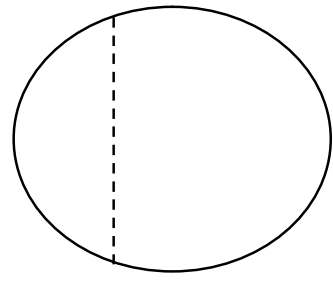

Figure 2. Opener of the lid.

3. Glue it back with a duct tape.

4. Glue the servo at the near edge of the cut, and use a string to be put into the opener, using a concept of push and pull by the servo (see Figure 3).

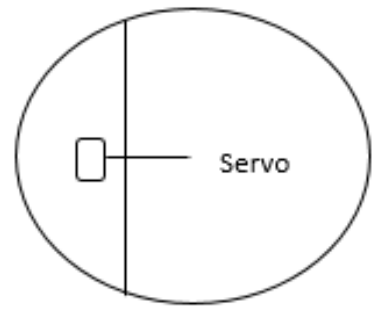

Figure 3. Servo attachment to the lid

5. Next, glue the opener to the top of the dustbin.

How the servo is attached to the lid of the bin needs to also be considered carefully. The servo should be able to freely lift the lid. One may use copper to connect servo to the lid, but it is prone to breaking. Therefore, this project simply uses a light string as seen in Figure 4.

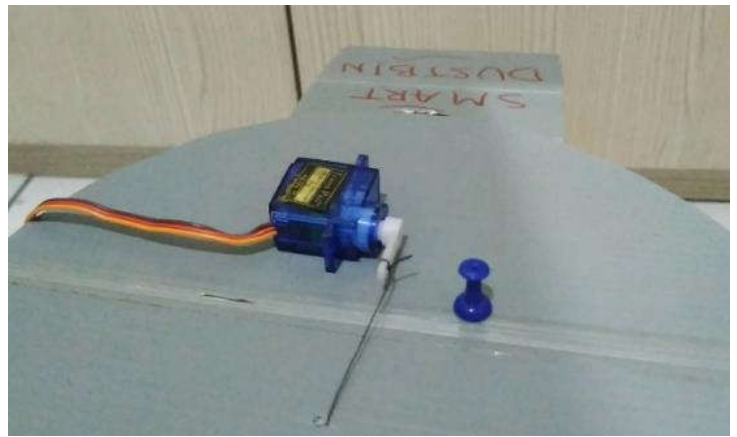

Figure 4. Placement of Servo motor on the lid. To open and close the lid, the servo is connected to the moving lid using a string.

The final result of the dustbin is shown in Figure 5. The wiring of the circuit is placed inside a box behind the bin to not disturb the operation of the bin.

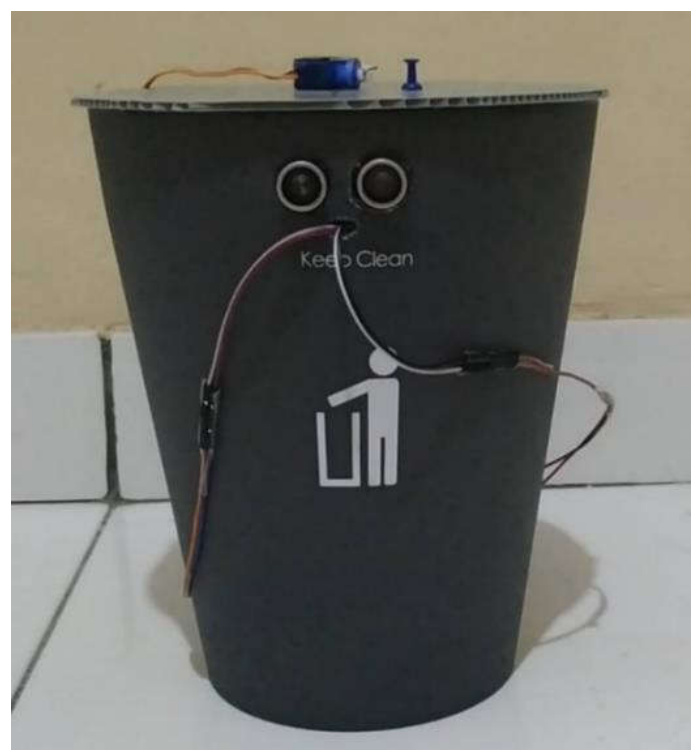

Figure 5. Appearance of Smart Dustbin Prototype. The two eyes-like protrusion is the ultrasonic sensor to detect user's hand 


\section{The Operation}

The automatic dustbin is triggered when the object distance to the ultrasonic sensor outside reads less than 15 $\mathrm{cm}$, which is interpreted as someone's hand trying to approach the bin. The servo then is commanded by the microcontroller to open the trash cover. The lid will open for a few seconds and then immediately shut again. Here's in more depth how it works:

1. With a maximum distance of $15 \mathrm{~cm}$, the ultrasonic sensor in the front will detect the object in front of it.

2. That processes the trigger signal to drive the servo, after which the signal is sent to Arduino as processing information.

3. This is where the servo's primary function is to open and close the trash bin depending on the signal and time of the trigger sensor.

4. Conditions will be checked in the bin after the closing process, whether or not the condition is in complete condition.

5. The buzzer will sound if the trash bin is full (the height of the trash within has reached ultrasonic).

The operational instruction to use the device is as follows:

1. Put your hand in front of the sensor.

2. When your trash is detected by the sensor, the lid will open and you can throw away your trash

3. After you thrown the trash, don't forget to keep your hand away from the sensor or the lid won't stop to open.

4. When the trash is full, the other sensor will detect the trash level inside and it will create a buzzer.

Empty the trash and the buzzer will stop.

\section{Software Design}

The code to control and manage the ultrasonic sensors and servo for this project is shown in Table 4.

\section{Table 4. Arduino Code}

\#include <Servo.h $>$

\#include $<$ UltraDistSensor.h $>$

Servo servo;

UltraDistSensor mysensor;

UltraDistSensor mysensor2;

int buzzpin = 13;

float reading, reading2;

void $\operatorname{setup}()\{$

servo.attach(11);

mysensor.attach(10,9); //trigger, echo servo

mysensor 2.attach $(4,3)$; //trigger, echo buzzer

pinMode(buzzpin, OUTPUT); \} void $\operatorname{loop}()\{$

reading $=$ mysensor.distance $\operatorname{InCm}()$;

reading $2=$ mysensor2.distance $\operatorname{InCm}()$;

if(reading $<15$ )

\{

servo.write(0); // Turn Servo to 0

delay(2000);

\}

else \{

servo.write(180); //Turn it 180

$\operatorname{delay}(50) ;\}$

delayMicroseconds(2);

if (reading $2<=11$ )

\{

digitalWrite(buzzpin, HIGH);

\}

else

\{

digitalWrite(buzzpin, LOW);

\}

$\operatorname{delay}(30) ;$

\}

Line 17 to line 21 in the code depicts the process when the servo opens the lid. The distance threshold is set to be 15 $\mathrm{cm}$. This number could be adjusted as necessary.

Line 26 to line 33 depicts the process of buzzer sounding the alarm when there's object (trash inside the bin) causing the sensor unable to read the other side of the bin. This number could also be adjusted depending on the circumference of the bin.

\section{Result}

In order to ensure that the bin is properly working, there are two processes to be tested.

1. The bin could open automatically when a hand is approaching the sensor on the outside of the bin. After a few seconds (giving time for the user to throw the trash into the bin) the bin should close automatically.

2. The alarm should sound if the bin is full and should stop sounding when the trash is emptied.

These two tests are successful as seen in Figure 6.

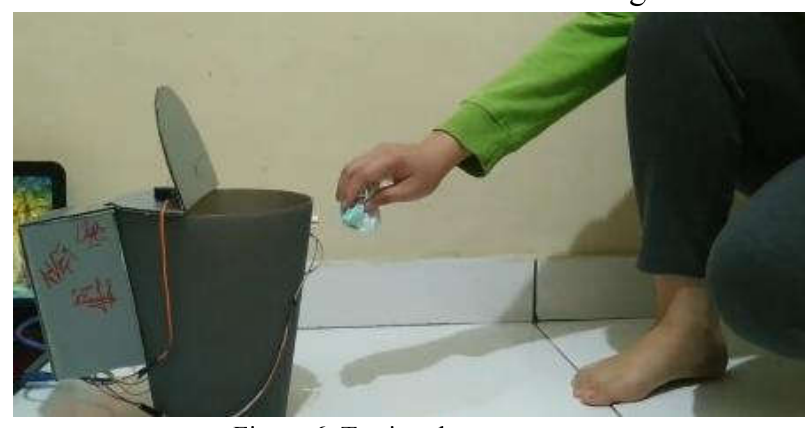

Figure 6. Testing the prototype 


\section{CONCLUSION}

Technology and technologies are not going to go any other way than to make progress. Through inventing new tools and materials, we could construct so many things just by combining a few components that are confined to our imaginations. This project is one example of how to demonstrate that by using simple sensors and some basic materials, we can create a product that is easy yet that can help to make people more comfortable.

Considering from all the components that are designed in a simple way to make a smart trash that is interactive and unique, which can attract the interest of people around to dispose of waste in its place and help protect the environment without fear of getting dirty.

There is still room for improvement with this project, but we hope that this project will inspire people and make them aware that you do not need advanced skills or understanding to be able to create something with your own hands and basic knowledge for a better future of technology.

\section{REFERENCES}

[1] How to drive two ultrasonic sensors, 2017, [online] Available: https://stackoverflow.com/questions/2582 3179/ driving-two-ultrasonic-sensor-with-arduino (Accessed: 25 August 2017).

[2] Interfacing buzzer, 2017, [online] Available: https://www.instructables.com/id/Interfacing-Buzzer-toArduino/ (Accessed: 9 September 2017).

[3] Detecting Distance Using Ultrasonic Sensor, 2017, [online] Available: https://www.hackster.io/arbazhussa in/distance-calculation-with-ultrasonic-sensor-26d63e (Accessed: 12 September 2017). 